\title{
STRUCTURE OF THE HYADES CLUSTER
}

\author{
G. PELS, J. H. OORT, and H. A. PELS-K LUYVER
}

Sterrewacht, Leiden, The Netherlands

H. A. Pels and J. H. Oort have discussed the results of a search for fainter Hyades that had been made by G. Pels. The search extended over a region of about $15^{\circ}$ radius around the Hyades centre. It was made from proper motions determined from a comparison of Astrographic Catalogue positions with those on plates taken with the $34-\mathrm{cm}$ Leiden refractor. The data extend to about $13^{m}$. In part of the field comparisons were also made with plates taken for the Astrographic Charts, in order to extend the luminosity distribution to somewhat fainter magnitudes.

The survey has roughly doubled the number of known Hyades. A combination of the present survey with that of Van Bueren gave a regular distribution on the sky with no observable deviation from circular symmetry.

Using a plausible extension of the luminosity function for fainter members an approximate mass density distribution was computed. Using the corresponding gravitational field it was shown that the observed space distribution of the cluster members agrees with that expected for a cluster in dynamical equilibrium, having a Maxwellian velocity distribution with a cut-off corresponding with the outer limit of the cluster. The average velocity in one coordinate is about $\pm 0.2 \mathrm{~km} \mathrm{~s}^{-1}$. The age of the cluster is indeed such that it should have reached approximate equilibrium.

There is a distinct segregation of stars of different mass.

The problem of outlying members is still under investigation.

A full account has been submitted for publication in Astronomy and Astrophysics. 